\begin{tabular}{|c|c|}
\hline Title & Development of a 3D collagen scaffold coated with multiwalled carbon nanotubes \\
\hline Author(s) & Hirata, Eri; Uo, Motohiro; Takita, Hiroko; A kasaka, Tsukasa; W atari, Fumio; Y okoyama, A tsuro \\
\hline Citation & $\begin{array}{l}\text { Journal of Biomedical Materials Research. Part B, A pplied Biomaterials, 90b(2), 629-634 } \\
\text { https://doi.org/10.1002 jbm.b.31327 }\end{array}$ \\
\hline Issue Date & $2009-08$ \\
\hline Doc URL & http:/hdl .handle.net $/ 2115 / 38894$ \\
\hline Rights & $\begin{array}{l}\text { This is the pre peer reviewed version of the following article: Journal of Biomedical Materials Research, Part B, } \\
90 \mathrm{~B}(2), 2009 \text {, pp.629-634, which has been published in final form at http://dx.doi.org/10.1002/jbm.b.31327 }\end{array}$ \\
\hline Type & article (author version) \\
\hline File Information & Hirata_JBMR2009B_HUSCA P.pdf \\
\hline
\end{tabular}

Instructions for use 


\title{
Development of a 3D collagen scaffold coated with multiwalled carbon nanotubes
}

\author{
Eri Hirata, Motohiro Uo, Hiroko Takita, \\ Tsukasa Akasaka, Fumio Watari, Atsuro Yokoyama
}

Department of Oral Functional Science

Graduate School of Dental Medicine, Hokkaido University

*Address of corresponding author

Eri Hirata

Department of Oral Functional Science

Graduate School of Dental Medicine, Hokkaido University

Kita 13, Nishi 7, Kita-ku, Sappro 060-8586

Telephone: +81-11-706-4270 


\begin{abstract}
Carbon nanotubes (CNTs) have attractive biochemical properties such as strong cell adhesion and protein absorption, which are very useful for a cell cultivation scaffold. In this study, we prepared a multiwalled carbon nanotube-coated collagen sponge (MWCNT-coated sponge) to improve the surface properties of the collagen sponge, and its cell culturing properties were examined. The suface of the collagen sponge was homogeneously coated with MWCNTs by dispersion. MC3T3-E1 cells were cultured on and inside the MWCNT-coated sponge. The DNA content on the MWCNT-coated sponge after 1 week of culture was significantly higher than on an uncoated collagen sponge $(p<0.05)$. There was no significant difference between the estimated ALP activity normalized by DNA quantity on the MWCNT-coated sponge and that on the uncoated collagen sponge which is well known as one of the best scaffolds for cell cultivation. In addition, the MWCNT-coated surface shows strong cell adhesion. Therefore, the MWCNT-coated collagen sponge is expected to be a useful 3D scaffold for cell cultivation.
\end{abstract}

Keywords: carbon nanotubes, coating, collagen sponge honeycomb, 3D scaffolds,

cell culture 


\section{Introduction}

Carbon nanotubes (CNTs) have high chemical stability, as well as biochemical properties such as strong cell adhesion ${ }^{1)}$, protein absorption ${ }^{2)}$ and cell differentiation ${ }^{3)}$. Favorable biocompatibility in vivo was also reported ${ }^{4)}$. Therefore, CNTs have been employed for use as a cell culture scaffold in the form of CNT sheets ${ }^{2)}$ and in composite with collagen $^{5)}$ or polymers ${ }^{6)}$.

On the other hand, for regeneration of more complicated tissues such as bone, a three-dimensional (3D) scaffold is needed. The required properties in addition to the 3D scaffold are biocompatibility, 3D cell proliferation and cell differentiation. Thus, CNTs would be useful as the substrate of a 3D scaffold. Currently, collagen sponges are widely used as 3D scaffolds because of their high biocompatibility. Therefore, CNT coating on the collagen sponge surface could improve its utility as a 3D scaffold.

In this study, multiwalled carbon nanotubes (MWCNTs) were used to coat the surface of a collagen sponge honeycomb to improve its surface properties, and the cell attachment and proliferation in the early term were estimated.

\section{Experimental procedure}

(1) Preparation of MWCNT-coated sponge

Multiwalled carbon nanotubes (MWCNTs; 20 50nm in diameter, CNTs Co.Ltd., Korea) were carboxylated by the reported method 7 ) and dispersed in $1 \mathrm{w} / \mathrm{v} \%$ sodium cholate solution to form a 100ppm MWCNT suspension. A collagen sponge honeycomb (AteloCell $^{\mathbb{B}} ; 3 \times 3 \times 2 \mathrm{~mm}$ in size, pore size $200 \sim 400 \mu \mathrm{m}$, Koken Co.,Ltd, Japan) was soaked in the MWCNT suspension for 1 hour under gentle shaking and then rinsed with phosphate-buffered saline(PBS; Gibco, U.S.A.). The obtained MWCNT-coated collagen sponge (MWCNT-coated sponge) was observed by scanning electron microscopy(SEM; S4000, Hitachi, Japan). The specific resistance of each scaffold was also measured using a digital multimeter (type175, Fluke, USA).

(2) Cell culture 
Mouse osteoblast MC3T3-E1 cells were suspended at 3.0x10 ${ }^{4}$ cells $/ \mathrm{mL}$ in minimum essential medium, alpha modification ( $\alpha$-MEM; Gibco, U.S.A), containing $10 \%$ fetal bovine serum (FBS; Biowest, U.S.A.) and 1\% penicillin/streptomycin. Collagen and MWCNT-coated collagen sponges were placed in the wells of a 96-well plate, and $50 \mu \mathrm{l}$ of the cell suspension was seeded on scaffold in the wells and then incubated for 7,14 or 21 days at $37^{\circ} \mathrm{C}$ in a $5 \% \mathrm{CO}_{2}$ atmosphere.

(3) Cell morphology

After 7 days of cell culture, the samples were examined biochemically and observed by SEM and optical microscopy. The samples were washed twice with PBS and fixed with $2 \%$ glutaraldehyde. After dehydration through a graded series of water-ethanol, half of the samples were freeze-dried and sputter-coated with palladium-platinum for SEM observation. The others were embedded in paraffin and HE-stained for optical microscopic observation. The cell surface morphology was observed by SEM. The cell proliferation on the internal part of the scaffold was observed by optical microscopy.

(4) DNA quantification and ALP activity

After 14 or 21 days of cell culture, the amounts of DNA and ALP activities of the samples were measured. The samples were washed twice with PBS. One hundred microliters of the cell suspension containing 0.2\% IGEPAL CA-630 (Sigma-Aldrich Co.), $10 \mathrm{mM}$ Tris- $\mathrm{HCl}$ and $1 \mathrm{mM} \mathrm{MgCl}_{2}, \mathrm{pH} 7.4$ was added to each sample. The samples were frozen, thawed and homogenized. The sample solution was then shaken for 30 minutes and centrifuged.

Picogreen (Molecular Probes, Leiden, The Netherlands) was used to measure DNA content. Thirty microliters of the supernatant fluid in the sample gathered with $70 \mu \mathrm{l}$ of TE buffer was added to $100 \mathrm{ml}$ of picogreen working solution and incubated for $10 \mathrm{~min}$ at room temperature in the dark. After incubation, DNA was measured using a fluorometer (F-3000, Hitachi, Japan) with the excitation filter set at 365nm and the emission filter at 450nm. A DNA standard curve was made with Lamda DNA. 
Alkaline phosphatase (ALP) activity was measured with LabAssay (Wako, Japan). Twenty microliters of the supernatant fluid in the sample was added to $100 \mu \mathrm{l}$ of $p$-nitrophenol phosphate in carbonate buffer and incubated for 15 minutes at $37^{\circ} \mathrm{C}$. After $80 \mu \mathrm{l}$ of $\mathrm{NaOH}$ was added, absorbance was measured at $405 \mathrm{~nm}$ using a plate analyzer (ETY-300, TOYO, Japan) and enzyme activity was determined from the calibration curve of the $p$-nitrophenol standard. ALP activity was normalized by DNA content.

DNA content and ALP activity were determined in 4 samples and expressed as means \pm standard deviations. Data for these measurements were analyzed with Student's two-tailed $t$-test, assuming equal variance, using Microsoft Excel. Statistically significant values were defined as $p<0.05$.

\section{Results}

(1) Scaffold morphology

Figure 1 shows the shape and the upper surface of a collagen sponge honeycomb. The pore size of the scaffold was $100-400 \mu \mathrm{m}$ and the pores were longitudinally connected from the top to the bottom surfaces. The surface structure was smooth.

Figure 2 shows the shape and the surface of an MWCNT-coated sponge. The whole shape and honeycomb structure of the collagen sponge were stable after MWCNT coating, as shown in Figures 2a and 2c. Figure 2b shows the raw MWCNTs. The sponge surface was homogeneously covered by MWCNTs as shown in Figure 2d and no aggregation of MWCNTs was observed. The MWCNT-coated sponge showed electron conductivity and the specific resistance was estimated to be $8 \times 10^{3} \Omega \mathrm{m}$, but the resistance of the collagen sponge could not be measured because it was over the detection limit $(40 \mathrm{M} \Omega)$ of the digital multimeter.

(2) Cell morphology

Figure 3 shows optical microscope images after 1-week incubation. The HE-stained paraffin-embedded specimens were sliced as shown in 3a. The cells covered and spread on the internal sidewall of the CNT-coated sponge as shown in Figures 3c and 3d, 
whereas few cells were observed in the collagen sponge shown in Figure 3b. Figure 4 shows SEM images of the cells after 1-week incubation. The cells attached to the surfaces of both scaffolds as shown in Figure 4a and 4c. However, at higher magnification, the filopodia on the MWCNT net shown in Figure 4d were distinct from those on the collagen sponge shown in Figure 4b.

(3) Cell proliferation and differentiation

The DNA content and ALP activity of the cells cultivated for 14 or 21 days on the sponges are shown in Figure 5. After 7 days of culture the ALP activities of the cells on both scaffolds were lower than the detection limit of the assay kit used. The DNA content in the cells attached to the MWCNT-coated sponges was significantly higher than that on the uncoated collagen sponges $(p<0.05)$. There was no significant difference between the scaffolds for ALP activity and ALP activity normalized by DNA content at $\mathrm{p}>0.1$. 


\section{Discussion}

In this study, a surface-modified collagen sponge using MWCNTs was prepared. The surface of the MWCNT-coated sponge was homogeneously covered with MWCNTs using carboxylated MWCNT dispersion with a surfactant. In Fig. 3c, it can be seen that a large number of cells attached to the internal sidewall of the collagen sponge. The sidewall of the collagen sponge is vertical; thus, it is normally difficult for cells to attach to it. However, MWCNT coating increased the initial cell attachment to the sidewall. One of the reasons for the high cell attachment was suggested to be the mechanical contact between cells and MWCNTs, as can be seen in Fig. 4d, which shows the filopodia of a cell that appear to be twisted around the MWCNTs.

The DNA content of the cells attached to the CNT-coated sponge was significantly higher than that on the uncoated sponge. Usually, DNA content is in proportion to the cell population. Thus, MWCNT coating improved the initial cell attachment to the collagen sponge and assisted proliferation during culture. In a previous study, one of the authors demonstrated that cell growth, proliferation and absorption of the protein in serum on a CNT sheet were higher than on a graphite sheet ${ }^{2)}$. Higher protein adsorption on carboxylated MWCNTs than on non-treated MWCNTs ${ }^{10)}$ were also reported. In addition, the surface roughness of the sponge was increased with MWCNTs as shown in Fig.1 and 2. Therefore, the increase in the surface roughness and the adsorption property of the MWCNT coated surface would be effective for the initial cell attachment.

In this study, the collagen sponge honeycomb, which has many straight macropores $(\sim 500 \mu \mathrm{m})$ and good mechanical strength ${ }^{9}$. was coated with MWCNTs and good osteoblast cell attachment and proliferation were obtained. Shi et al. developed a CNT-based porous scaffold with pore sizes of $80-100 \mu \mathrm{m}$ from a composite of SWCNTs and a polymer, and good cell attachment and proliferation were demonstrated ${ }^{6}$. However, to employ the 3D scaffold, interconnecting macropores are required to obtain cell proliferation toward the internal pores. The MWCNT-coating on the collagen sponge honeycomb improved the cell attachment on the internal pore surface as shown in Fig.3, then, the homogeneous cell proliferation on both internal and external surface of the MWCNT coated sponge was achieved. In our previous study, new bone formation on the MWCNTs sintered monolith was reported. Furthermore, the MWCNT-coated sponge exhibited electrical conductivity, whereas collagen is 
nonconductive; thus, MWCNT coating could make possible culture under electrical stimulation. It has already been reported that the stimulation of osteoblasts by electric pulses can accelerate the multistep process of biomineralization in vitro ${ }^{8)}$. Therefore, the MWCNT-coated sponge would suggest the feasibility of its use as a scaffold for bone regeneration.

\section{Conclusion}

A 3D cell culture scaffold was obtained by homogeneously coating a collagen sponge honeycomb with MWCNTs. The cell attachment to the internal pore surface was improved by the MWCNT coating. Thereby, homogeneous cell proliferation was achieved not only on the sponge face but also on the internal pore surface.

Therefore, the MWCNT-coated collagen sponge is advantageous as a 3D scaffold for cell cultivation and tissue engineering.

\section{Acknowledgements}

This work was supported by a Grant-in Aid for Basic Research (B) 19390488 from the Ministry of Education, Science, Culture and Sport of Japan. 


\section{References}

1. Aoki N, Yokoyama A, Nodasaka Y, Akasaka T, Uo M, Sato Y, Tohji K, Watari F. Strikingly Extended Morphology of Cells Grown on Carbon Nanotubes. Chem Lett 2006;35:508-509.

2. Aoki N, Akasaka T, Watari F, Yokoyama A. Carbon Nanotubes as Scaffolds for Cell Culture and Effect on Cellular Functions. Dental Material Journal 2007;26:178-185

3. Zanello LP, Zhao B, Hu H, Haddon RC. Bone Cell Proliferation on Carbon Nanotubes. Nano Lett 2006;6:562-567.

4. Yokoyama A, Sato Y, Nodasaka Y, Yamamoto S, Kawasaki T, Shindoh M, Kohgo T, Akasaka T, Uo M, Watari F, Tohji K. Biological Behavior of Hat-Stacked Carbon Nanofibers in the Subcutaneous Tissue in Rats. Nano Lett 2005;5:157-161.

5. MacDonald RA, Laurenzi F, Viswanathan G, Ajayan PM, Stegemann JP. Collagen-carbon nanotube composite materials as scaffolds in tissue engineering. $\mathrm{J}$ Biomed Mater Res A 2005;74A:489-496.

6. Shi X, Sitharaman B, Pham QP, Liang F, Wu K, Billup WE, Wilson LJ, Mikos AG, Fabrication of porous ultra-short single-walled carbon nanotube nanocomposite scaffolds for bone tissue engineering. Biomaterials 2007;28:4078-4090.

7. Peng H, Alemany LB, Margrave JL, Khabashesku VN. Sidewall Carboxylic Acid Functionalization of Single-Walled Carbon Nanotubes. J Am Chem Soc 2003;125: 15174-15181.

8. Wiesmann H, Hartig M, Stratmann U, Meyer U, Joos U. Electrical stimulation influences of osteoblast-like cells in vitro. Biochimica et Biophysica Acta 2001;1538:28-37

9. Kakudo N, Shimotsuma A, Miyake S, Kushida S, Kusumoto K. Bone Tissue engineering using human adipose-derived stem cells and honeycomb collagen scaffold. J Biomed Mater Res A. 2008;84A:191-197.

10. Xaojun Li, Wei Chen, Qiwen Zhan, Liming Dai. Direct Mesurement of Interactions between Polypeptides and Carbon Nanotubes. J. Phys. Chem. B 2006; 110: 12621-12625 

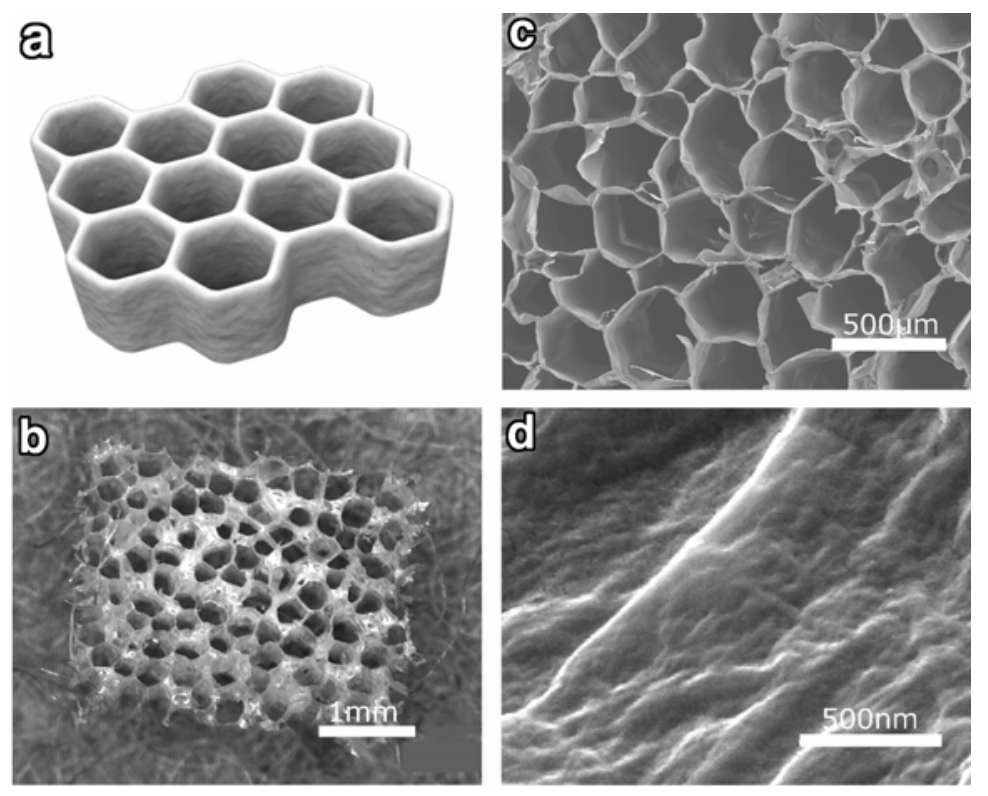

Figure 1. The collagen sponge honeycomb: (a) a schematic model, (b) the whole shape of the collagen sponge, (c)-(d) SEM images of the collagen sponge, (c) at lower magnification, (d) at higher magnification.
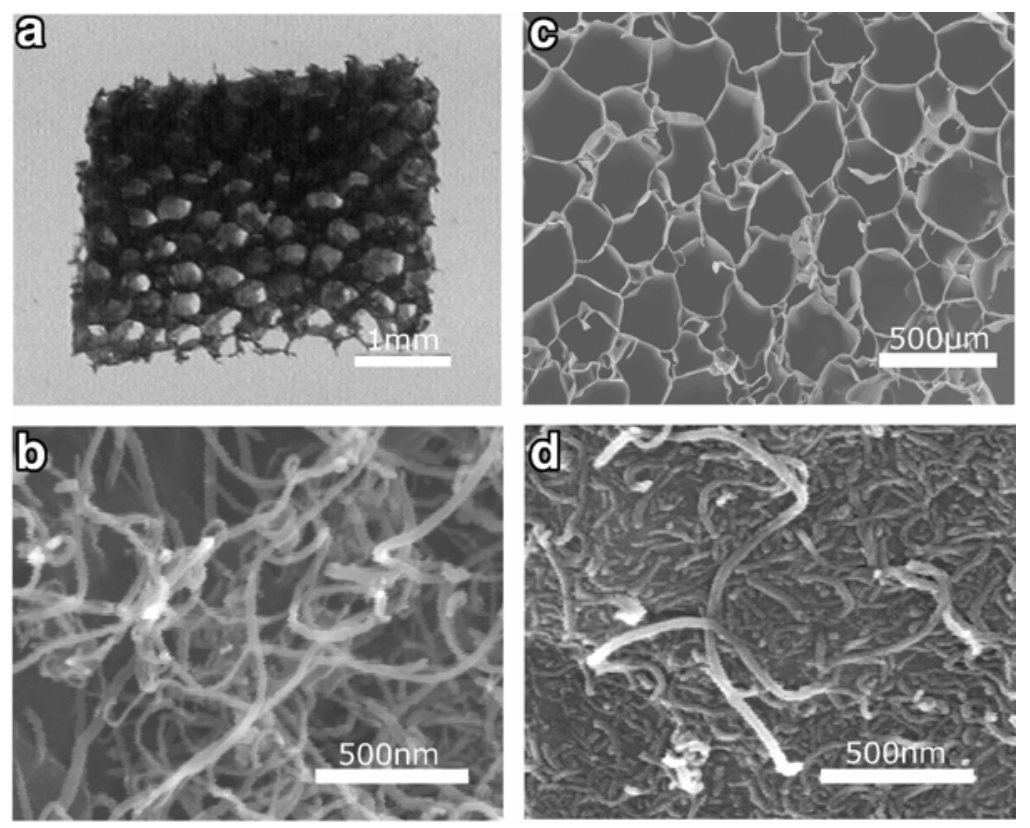

Figure 2. The MWCNT-coated sponge: (a) the whole shape of the MWCNT-coated sponge, (b) SEM image of untreated MWCNTs, (c)-(d) SEM images of the MWCNT-coated sponge, (c) at lower magnification, (d) at higher magnification. 


\section{อ}
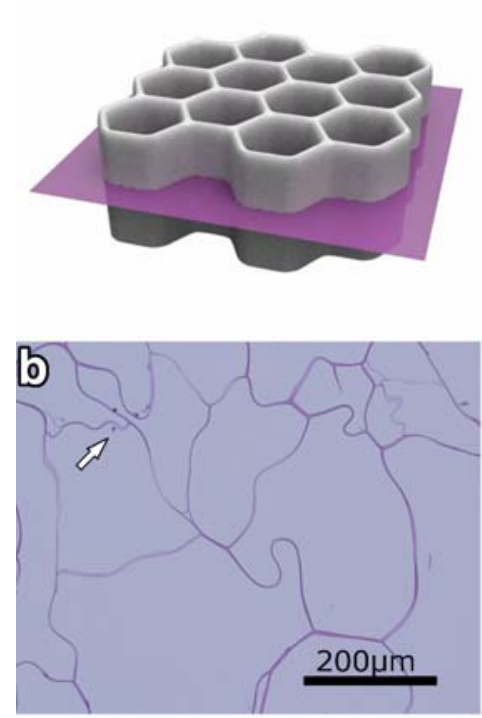
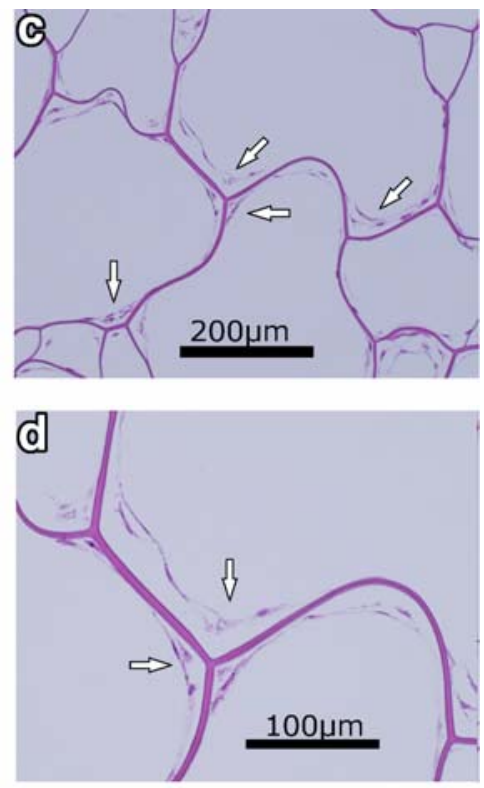

Figure 3. Optical microscopic observation after 1 week of culture: (a) paraffin-embedded specimens were sliced as in the schematic model (b) Few cells are observed in the collagen sponge. (c) Many cells are observed in the MWCNT-coated sponge at low magnification. (d) The cells spread on the sidewall of the MWCNT-coated sponge. 

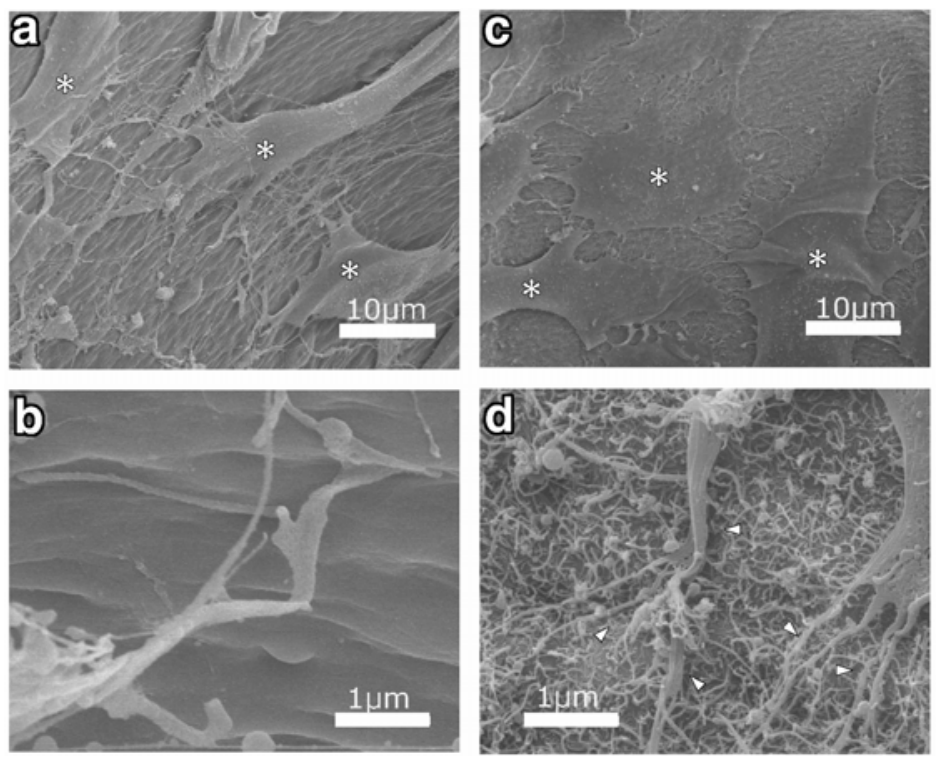

Figure 4. SEM observation after 1-week incubation: cells (asterisk) grown on the (a) collagen sponge and (c) MWCNT-coated sponge. SEM images at higher magnification: (b) collagen sponge, (d) cytoplasmic elongations (arrowhead) intertwined with MWCNTs on the surface of the MWCNT-coated sponge.

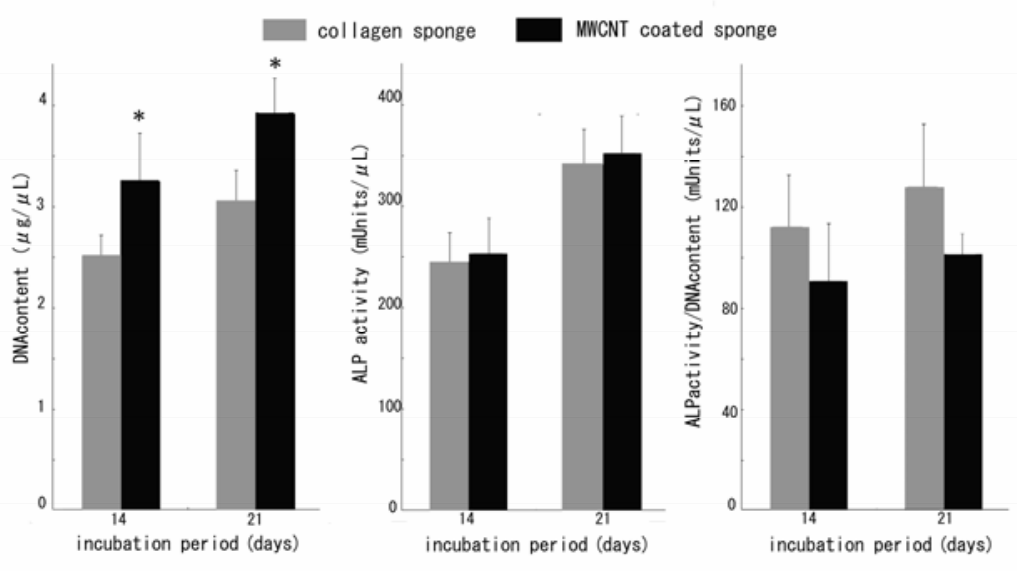

Figure 5. DNA content (left) of the cells cultured on the collagen sponges and MWCNT-coated sponges for 2 and 3 weeks; $n=4$. The asterisk indicates a statistically significant difference between two scaffold groups (t-test: $p<0.05)$. There were no significant differences between the scaffolds for ALP activity (middle) and ALP/DNA (right). 